This article was published in Polymer Engineering and Science, 56(5), 590-597, 2016

http://dx.doi.org/10.1002/pen.24284

\title{
Synthesis of Multihollow Polyester Particles in Supra- and Infra-Millimeter Size Ranges by Double EmulsionProcess
}

\author{
Sandra Monteiro, ${ }^{1}$ Ângela Dias, ${ }^{1}$ Jorge Martins, ${ }^{1,2}$ Luısa Carvalho, ${ }^{1,2}$ Fernão \\ D. Magalhães 1 \\ ${ }^{1}$ LEPABE - Faculdade de Engenharia, Universidade do Porto, Rua Dr. Porto, Roberto \\ Frias 4200-465, Portugal \\ 2 DEMAD - Department of Wood Engineering, Polytechnic Institute of Viseu, Campus \\ Politecnico De Repeses, Viseu 3504-510, Portugal
}

Multivesiculated particles (MVPs) are obtained from a double emulsion system, being currently used as opacifying agents by waterborne paint industry. They have high mechanical resistance and low density as a result of a rigid multialveolar structure formed by cross-linked poly- ester. Depending on the particle sizes, different novel applications can be envisaged. This work studies how the conventional one-step batch emulsification approach can be adapted for producing MVPs with average diameters ranging from tens of microns to a few millimeters. The Taguchi method was employed to identify the level of influence of several process variables on the final yield of small $(<100 \mu \mathrm{m})$ or large ( $>1 \mathrm{~mm}$ ) particles. Poly(vinyl alcohol) (PVA) concentration and molecular weight proved to be the most significant factors to obtain small particles. For large particles, the most significant factors were the PVA molecular weight and the stirring rate during the curing phase. Uniform vesiculation and low particle density could only be obtained by adjusting the stirring conditions during dispersion of the organic phase, due to limitations in water diffusion rate into large organic droplets. Large particles exhibited tendency to fracture due to residual stresses, but this problem was solved by using an organic-soluble cure initiator.

\section{INTRODUCTION}

Multivesiculated particles (MVPs) are a type of multihollow polymeric particles that have industrial use as opacifying agents in waterborne paints. The large refractive index difference between polymer shell and entrapped air results in scattering of incident light, increasing the opacity of a coating loaded with these particles. MVPs can therefore partially replace the costly titanium dioxide pigment traditionally used [1].

MVPs are produced by a double emulsion process that yields crosslinked polyester particles 
with diameters between 1 and $10 \mu \mathrm{m}$ [1-3]. Their interior consists of multiple homogeneously distributed spherical microalveoli separated by thin polymer walls. Considering the mechanical resistance of the thermoset matrix combined with the multivesiculated internal morphology, other applications beyond the context of coating opacification can be envisaged for these particles [4]. This, however, implies producing them with larger sizes, depending on the particular application. Particles with diameters of tens of microns may have uses in encapsulation of active substances $[5,6]$, low-density fillers in thermoplastic [7], and thermoset matrixes [8] and syntactic foams [9]. Larger diameters, in the range of a few millimeters, may be used as low-density fillers in particleboards [10] or as catalyst support [11].

This work studies the feasibility of producing MVPs with different size ranges, using as reference the procedure used for synthesis of the "small" MVPs used in paint formulations. This approach [1] starts with dispersion of an organic phase, comprising unsaturated polyester dissolved in styrene, in an aqueous solution of poly(vinyl alcohol) (PVA) at high stirring rate (dispersion step). Water diffuses into the dispersed organic phase, forming droplets in its interior. This water-in-oil (W/O) emulsion is stabilized by hydrophilic acid- base ion-pairs formed by neutralization of terminal polyester carboxylic groups with an added amine, without need for addition of a surfactant. The whole system is, therefore, a water-in-oil-in-water $(\mathrm{W} / \mathrm{O} / \mathrm{W})$ double emulsion. The organic phase is then hardened by addition of a radical initiator (cure step). The entrapped water evaporates and originates air-filled vesicles after drying. Figure 1 illustrates the entire synthesis process.

In a previous work, we studied the influence of different factors on MVPs synthesis, namely the time of dispersion of the organic phase in the aqueous medium, and the concentrations of amine, polyester, and protective colloids [1]. The role of the type of the amine in the stabilization of the W/O emulsion sys- tem has also been studied [2], as well as the effect of curing conditions on the particles properties [3]. All these works dealt with average diameters below $10 \mu \mathrm{m}$. Larger sizes, in the order of hundreds of micron, have been produced only using a micro- fluidic system, using a two-step emulsification approach distinct from the one-step batch reactor route described above [12].

This work discusses the influence of several process variables and procedures on the size and internal vesiculation of MVPs. It is shown that homogeneously vesiculated MVPs can be produced in a batch reactor using a double-emulsion approach, with average diameters ranging from tens of microns to a few millimeters.

\section{EXPERIMENTAL}

Materials

The unsaturated polyester - obtained by polymerization of maleic anhydride, phtalic anhydride, and propylene glycol monomers - was kindly provided by Resiquímica (Mem Martins, Portugal) in the form of a solution in styrene (weight ratio 70/30, acid value 15-20 mg KOH/g and Brookfield viscosity in the range of 1000-2000 $\mathrm{mPa} \mathrm{s}$ ). Styrene and ammoniacal ferrous sulphate were also provided by Resiquímica. Poly (vinyl alcohol) (Mowiol 47-88, MW > 205,000 g/mol, degree of hydrolysis 5 $88 \%$ ) was kindly provided by Archroma (Reinach, Switzerland). Poly (vinyl alcohol) (MW 5 13,000 g/mol, degree of hydrolysis 5 88\%), diethylenetriamine (DETA, 98\%), and cumene hydroperoxide (technical grade, $80 \%$ ) were supplied by Sigma-Aldrich. Dibenzoyl peroxide $75 \%$ remainder water was provided by Acros Organics (Geel, Belgium). 


\section{Multivesiculated Particles Production}

The formulation of the single-step double emulsion is described in previous works $[1,13]$ and was adopted as the reference. The unsaturated polyester solution $(15 \mathrm{~g})$ was first neutralized with DETA $(0.21 \mathrm{~g})$ and then diluted in styrene $(6.5 \mathrm{~g})$. Then this mixture was gradually added to an aqueous solution (61.7 g), containing 2.3\% wt. PVA, in a jacketed glass reactor (100 ml), under constant mechanical agitation (600 rpm) using a helix impeller (40 mm diameter). After all organic phase being added, stirring was maintained for 20 min. The cross-linking of the unsaturated polyester with styrene was then initiated by adding $0.12 \mathrm{~g}$ of cumene hydroperoxide and $0.01 \mathrm{~g}$ of ferrous sulphate. The curing stage lasted $2 \mathrm{~h}$ at $70^{\circ} \mathrm{C}$. Final particles were washed with distilled water and dried at $80^{\circ} \mathrm{C}$ for $2 \mathrm{~h}$.

An alternative cure procedure was also studied, using dibenzoyl peroxide as curing initiator. The organic phase (polyester, styrene, and amine solution) was mixed with $3 \mathrm{wt} \%$ of dibenzoyl peroxide and cured at $60^{\circ} \mathrm{C}$ for $2 \mathrm{~h}$.

\section{Particle Size Distribution}

The particle size distribution (PSD) of the smaller MVPs $(<100 \mathrm{~mm})$ was measured on a Beckman Coulter LS230 light scattering system, with Polarization Intensity Differential Scattering (PIDS) assembly and an obscuration of $45 \%$. The particle size distributions were computed by the equipment's software, based on Mie theory for light scattering. The measurements were made after sample collection from the reactor and after particles drying.

Final particle size distributions for larger MVPs $(>1 \mathrm{~mm}$ ) were obtained by sieving (sieves with open mesh of 1 and $2 \mathrm{~mm}$, supplied by Retsch)

\section{Scanning Electron Microscopy}

Particle morphology and internal vesiculation were observed by scanning electron microscopy, using an FEI Quanta 400FEG ESEM/EDAX Genesis X4M equipment (SEM) analysis at Centro de Materiais da Universidade do Porto (CEMUP). For this purpose, dried particles (larger and smaller) were encapsulated in epoxy resin, and the resulting composite was fractured

in liquid nitrogen after hardening at $90^{\circ} \mathrm{C}$ overnight. Before being analyzed, samples were sputtered with gold/platinum using a K575X Sputter Coater by Quorum Technologies.

\section{Mercury Porosimetry}

The apparent density of the particles was determined by mercury porosimetry with a Poremaster 60 model from Quantachrome. The density of the polymer (nonvesiculated polyester reticulated with styrene) was determined by water pycnometry.

\section{Taguchi Method for Design of Experiments}

The effect of the following four independent process variables on particle size was studied: stirring rate during the dispersion step ( $v$ disp), stirring rate during the curing phase ( $v$ cure), PVA concentration, CPVA, and PVA molecular weight (MWPVA). Three levels were set for the first three variables and two for the last, as shown in Table 1.

The minimum number of experiments required by Taguchi method can be calculated based on the degrees of freedom (DOF) approach: $1+3 \times(3-1)+(2-1)=8$. Orthogonal arrays L9 or L18 could therefore be chosen for design of experiments. To improve robustness of results, L18 was chosen. For all experiments, the performance parameters were determined 
according to the weight of sieved particles for the different sizes considered. For all experiments, the MVPs production process was based on the reference procedure described before, using dibenzoyl peroxide as curing initiator.

\section{RESULTS AND DISCUSSION}

\section{Design of Experiments}

The complete Taguchi's experimental design is summarized in Table 2. Two distinct goals were defined. One consisted in obtaining particles with diameters smaller than $100 \mu \mathrm{m}$, appropriate for incorporation as fillers in thermoplastics or thermo- sets. The other implied obtaining diameters larger than $1 \mathrm{~mm}$, needed for incorporation in wood particleboards, for instance. Commercial statistical software package JMP was used for data analysis. To evaluate the significance level of the effects of the different factors, an analysis of variance (ANOVA) was performed.

\section{ANOVA Analysis}

The analysis of variance (ANOVA) is a powerful technique to explore the relative contribution of factors affecting a response. With ANOVA, the optimal combination of the process variables can be predicted. Figure 2 shows the prediction pro- files of the effects of the different factors on particle size. The effects of the several factors on each performance parameter (content of MVPs diameter $<100 \mu \mathrm{m}$ and $>1 \mathrm{~mm}$ ) are presented in Tables 3 and 4, respectively.

As shown in Table 3, ANOVA results reveal that the stirring rate during the dispersion step ( $v$ disp) has no significant effect in defining the particle size of MVPs. PVA concentration (CPVA) and molecular weight (MWPVA), on the other hand, are seen to be very significant for production of small particles. Concerning obtaining particles larger than $1 \mathrm{~mm}$ (Table 4), this is not affected by PVA concentration, while its molecular weight and the stirring rate during curing ( $v$ cure) can be considered very significant factors.

The fact that the stirring speed during dispersion of the organic phase in water does not have a relevant effect on the final particle sizes can be easily interpreted. The double emulsion droplets will tend to coalesce and form larger droplets if the stirring in the curing the step is lower than in the dispersion step. It is therefore in the initial stage of curing, while the organic phase is still sufficiently fluid, that the final particle sizes are defined, largely independently on the intensity of dispersion in the previous step. Stirring rate during cure, $v$ cure, is therefore a much more relevant factor for particle size, especially when large particles are the target. This is because shear tensions in the liquid medium must be minimized to allow for the dispersed phase to coalesce into sufficiently large droplets.

PVA acts as protective colloid, stabilizing the double emulsion droplets by adsorbing onto their surface [1]. It is understandable that its concentration has a major influence on the fraction of smaller particles, since small droplets can only sur- vive up to the final curing process if steric stabilization is effective. When large particles are intended, on the other hand, CPVA does not play a significant role, as the colloid concentration in the droplet surface is sparse and the droplet sizes are mainly controlled by shear rates.

The molecular weight of PVA showed to be a variable with a major influence. Increasing MWPVA simultaneously increases the fraction of small particles and decreases the fraction of large particles. On one hand, larger molecular weight originates significantly higher viscosity in the aqueous medium, increasing shear forces and causing rupture of larger organic droplets. On 
the other hand, it ensures more effective steric stabilization and reduces coalescence induced by droplet collision.

Figure 3 shows the examples of weight-based particle size distributions measured for Experiments 1, 5, 6 and 18. In the first case, a large mass fraction corresponds to particles with diameters above $1 \mathrm{~mm}$. Almost 30\%, however, still present sizes between 0.1 and $1 \mathrm{~mm}$. The way how these remaining smaller particles can be minimized will be discussed below. Experiments 5 and 6 yielded an intermediate distribution, as expected from the operation variables used. In Experiment 18, all variables have maximum values, except for vcure, and all particles fall within the small particle range, with sizes below $0.1 \mathrm{~mm}$.

The particle size distribution of the particles produced in Experiment 18 was determined in more detail using a light scattering system. The result is shown in Fig. 4. The distribution is relatively broad, with diameters in the range 5-55 $\mathrm{mm}$. A narrower distribution, also shown, was possible by using a $20 \mathrm{~mm}$ sieve. Figure 5a shows the SEM image of MVPs produced in Experiment 18, after sieving. As seen in Fig. 5b, the particles interior is uniformly vesiculated, with homogeneously distributed spherical alveoli separated by thin polymeric walls.

\section{Effect of Dispersion Stirring Rate on Particle Vesiculation and Density}

The previous section was focused on adjusting synthesis conditions for maximizing production of MVPs within an intended size range. However, in addition to particle diameter, the quality of internal vesiculation has also to be taken into consideration as this directly influences particle density and other properties like light diffraction/opacity. The formation of the internal alveoli occurs by water diffusion from the continuous aqueous phase toward the interior of the organic droplets, forming a double emulsion during the dispersion step. It is not a priori clear whether this process can effectively lead to uniform vesiculation of organic droplets with diameters above $100 \mu \mathrm{m}$.

SEM images shown in Fig. 6 illustrate the influence of different stirring rates during the dispersion step on internal particle morphology. For producing these particles, PVA concentration was $2.3 \mathrm{wt} \%$ and molecular weight $13,000 \mathrm{~g} / \mathrm{mol}$, cure temperature was $60^{\circ} \mathrm{C}$ during $2 \mathrm{~h}$, under a stirring rate of $200 \mathrm{rpm}$. The studied stirring rates during dispersion were $200,600,750,1000$, and $1400 \mathrm{rpm}$. As the stirring rate is increased from 200 to $750 \mathrm{rpm}$, the concentration of vesicles in the center of the particles visibly increases, maintaining an average diameter of about $2 \mathrm{~mm}$ (Fig. 6a-c). At $750 \mathrm{rpm}$, the particles are fully vesiculated. As the stirring rate is increased to $1000 \mathrm{rpm}$ (Fig. 6d), larger vesicles are observed, with diameters of about 10 $\mu \mathrm{m}$, but most are still within $2 \mu \mathrm{m}$ average size. At $1400 \mathrm{rpm}$, the particles remain fully vesiculated, but the alveoli are uniformly large, with about $15 \mu \mathrm{m}$ diameter. These results provide information on how the dispersion step determines particle vesiculation. The operation conditions used were the same as for Experiment 6 in the Taguchi method (Table 2), except that $v$ disp assumed a set of distinct values. As discussed before, the particles produced under these conditions have diameters in the order of several hundred microns. For organic phase droplets in this size range, water diffusion becomes a limiting step that significantly delays formation of the water-in-oil-in-water double emulsion and hence inner vesiculation. When stirring rate is kept low during this step, the droplets remain with relatively large sizes until hardening, and water penetration is not rapid enough to fully form a homogeneous double emulsion within the dispersed organic phase. Figure 7 shows the SEM image of the periphery of a cured particle produced under $v$ disp $=200 \mathrm{rpm}$. The concentration of vesicles is higher close to the 
external surface, forming a gradient relatable to the extent of water diffusion into the organic droplet. As rate of stirring is increased, the organic phase undergoes frequent rupture as a consequence of higher shear stresses, forming smaller droplets. Full emulsification of water within these droplets can therefore be effectively attained within the dispersion time for $v$ disp $=750 \mathrm{rpm}$. The droplet size ends up increasing again during the beginning of the curing step, where low stirring rates are used (200 rpm in this case), due to coalescence under lower shear. But the double emulsion is already fully established, and uniform vesiculation is achieved. If stirring is increased further, the droplet rupture/collision processes intensify, and coalescence of the water droplets emulsified within the organic phase occurs.

Larger vesicles start to form at $1000 \mathrm{rpm}$, and at $1400 \mathrm{rpm}$, most vesicles present large sizes of about $15 \mu \mathrm{m}$.

It can be concluded that the rate of stirring during the dispersion step, while not having a relevant effect on final particle size, has a major influence on the homogeneity of internal vesiculation, and on vesicle size.

The apparent density of the cured MVPs depends directly on vesiculation as this originates the air-filled void space within the dry particles. As expected, Fig. 8 shows that increasing stirring rate during dispersion leads to a reduction on apparent density. The effect is less significant for the higher stirring rates, since the particles are already fully vesiculated and only vesicle size increases. The lowest density of $0.52 \times 10^{3} \mathrm{~kg} / \mathrm{m}^{3}$ was obtained for $1400 \mathrm{rpm}$. Note that the density of the unvesiculated cross- linked polyester is $1.2 \times 10^{3} \mathrm{~kg} / \mathrm{m}^{3}$.

\section{Effect of the Type of Curing Initiator on Mechanical Stability of Large Particles}

The reference MVPs formulation uses cumene hydroperoxide as free radical initiator, in combination with a metal redox activator (aqueous solution of ferrous sulphate and diethylenetriamine), to promote cross-linking between polyester and styrene at relatively low temperatures [3]. This cure initiator system is added to the aqueous phase at the beginning of the curing step. However, when the larger particles were produced, with diameters above $1 \mathrm{~mm}$, it was observed that all became fractured during drying. This occurred even when gentle drying conditions were used, like $808 \mathrm{C}$ for $2 \mathrm{~h}$. Figure 9 shows the resulting cracked particles. This phenomenon can be attributed to evaporated water not being able to diffuse fast enough toward the exterior in large particles, rising internal pressure and causing fracture. Residual stresses, accumulated within the particle during polymer hardening, facilitate this type of structural failure. Since cure-initiating radicals are formed in the aqueous phase and then diffuse into the organic droplets, polymer hardening starts from the periphery toward the center of the particles. Therefore, the fact that the initiator is water soluble may actually contribute to development of residual stresses upon cure.

The former hypothesis was tested by replacing the original water-soluble initiator with dibenzoyl peroxide, BPO, which is directly soluble in the organic phase. BPO was therefore added to the polyester solution before its dispersion into the aqueous phase. Radical formation is in this case initiated by rising temperature (to $80^{\circ} \mathrm{C}$ or above), therefore preventing premature hardening before double emulsion formation. This implied investigating the appropriate $\mathrm{BPO}$ concentration in the polyester + styrene organic phase, in order to obtain a short curing time. Table 5 shows the gel time obtained for BPO concentrations of 1,2 , and $3 \mathrm{wt} \%$. For $3 \mathrm{wt} \%$, the organic solution begins to form a gel after about $5 \mathrm{~min}$. This concentration was tested in MVP production. The obtained MVPs were uniformly vesiculated and did not fracture upon 
drying in the same conditions as before.

\section{Effect of the Second Stirring Step During Dispersion on Particle Size}

As discussed in the section "ANOVA Analysis", the "optimal" operating conditions for producing large MVPs still yield a significant fraction of particles with smaller sizes (lower than $1 \mathrm{~mm}$ ). In an attempt to minimize this, the second dispersion step was introduced, in which the stirring speed was reduced to $200 \mathrm{rpm}$ for $15 \mathrm{~min}$ prior to initiating cure. Figure 10 shows the results obtained, for two initial dispersion speeds (750 and $1000 \mathrm{rpm})$. The lower stirring in the second step allows for coalescence of the organic droplets, leading to a significant shift of the final distribution toward the right. Interestingly, when stir- ring speed in the first step is increased to $1000 \mathrm{rpm}$, the final fraction of particles below $1 \mathrm{~mm}$ decreases even further. The higher initial shear in the aqueous medium produces a large number of smaller droplets that then coalesce into much larger ones when stirring decreases. Figure 11a shows the particles obtained using this new approach, while Fig. 11b shows the sur- face and interior of a particle fractured manually, showing the high degree of vesiculation.

\section{CONCLUSIONS}

Multivesiculated particles (MVPs) were successfully synthesized in two different size ranges $(<100 \mu \mathrm{m}$ and $>1 \mu \mathrm{m})$. The concentration and molecular weight of PVA, used as protective colloid, have a very significant influence in the production of small particles. MVPs with diameters below $100 \mu \mathrm{m}$ were obtained with PVA of 205,000 g/mol molecular weight and 3.45 wt $\%$ concentration.

Concerning production of large particles, the effect of some process variables was identified. Sufficiently high stirring rate during the dispersion of polyester droplets in water is essential to guarantee good vesiculation and thus minimum density. If organic droplet size is not reduced during this step, water is unable to diffuse into it to achieve full vesiculation. The yield of particles above $1 \mathrm{~mm}$ was determined mainly by PVA molecular weight and stirring rate during the curing step. This was further improved when a period of low stirring speed was added to the dispersion step, allowing for droplet coalescence before curing. Large particles with diameter above $1 \mathrm{~mm}$ and about $90 \mathrm{wt} \%$ yield was produced with PVA molecular weight of 13,000 $\mathrm{g} / \mathrm{mol}$, initial stirring in dispersion step were of $1000 \mathrm{rpm}$ for $20 \mathrm{~min}$ followed by $200 \mathrm{rpm}$ for $15 \mathrm{~min}$, and cur- ing under the same stirring rate for $2 \mathrm{~h}$.

The large particles fractured during drying due to water evaporation generating internal pressure build up. Residual stresses originated by polymer hardening being started at the outer shell and progressing toward the interior facilitated this tendency to fracture. This problem was eliminated by using an organic-soluble cure initiator instead of a water-soluble system.

\section{REFERENCES}

1. Â. Dias, J. Fidalgo, J. Machado, J. Moniz, A. Mendes, and F. Magalhães, Eur. Polym. J., 49, 664 (2013).

2. A. Dias, J. Machado, J. Moniz, A. Mendes, and F. Magalhães, Polym. Eng. Sci, 53, 2261 (2013).

3. A. Dias, J. Machado, J. Moniz, A. Mendes, and F. Magalhães, Polym. Eng. Sci., 54, 396 (2014). 
4. J. Fidalgo, A.M. Mendes, and F. Magalhães, Eur. Polym. J., 58, 173 (2014).

5. B. Peng and A. Imhof, Soft Matter, 11, 3589 (2015).

6. W. Wang, M.J. Zhang, and L. Chu, Acc. Chem. Res., 47, 373 (2014).

7. A. Doumbia, D. Jouannet, T. Falher, and L. Cauret, Key Eng. Mater., 859, 611 (2014).

8. H.S. Kim and M.A. Khamis, Compos. A, 32, 1311 (2001).

9. E. Wouterson, F. Boey, X. Hu, and S. Wong, Compos. Sci. Technol., 65, 1840 (2005).

10. A. Shalbafan, J. Luedtke, J. Welling, and H. Thoemen, Eur. J. Wood Wood Prod., 70, 287 (2012).

11. Z. Chen, M. Shi, C. Ma, Y. Chu, and A. Zhu, Powder Technol., 235, 467 (2013).

12. J. Fidalgo, A. Dias, A. Mendes, and F. Magalhães, Chem. Eng.J., 233, 323 (2013).,

13. J. Engelbrecht, D. Wet-Roos, A. Smit, and B. Cooray, U.S. Pat- ent 10/529764 (2006).

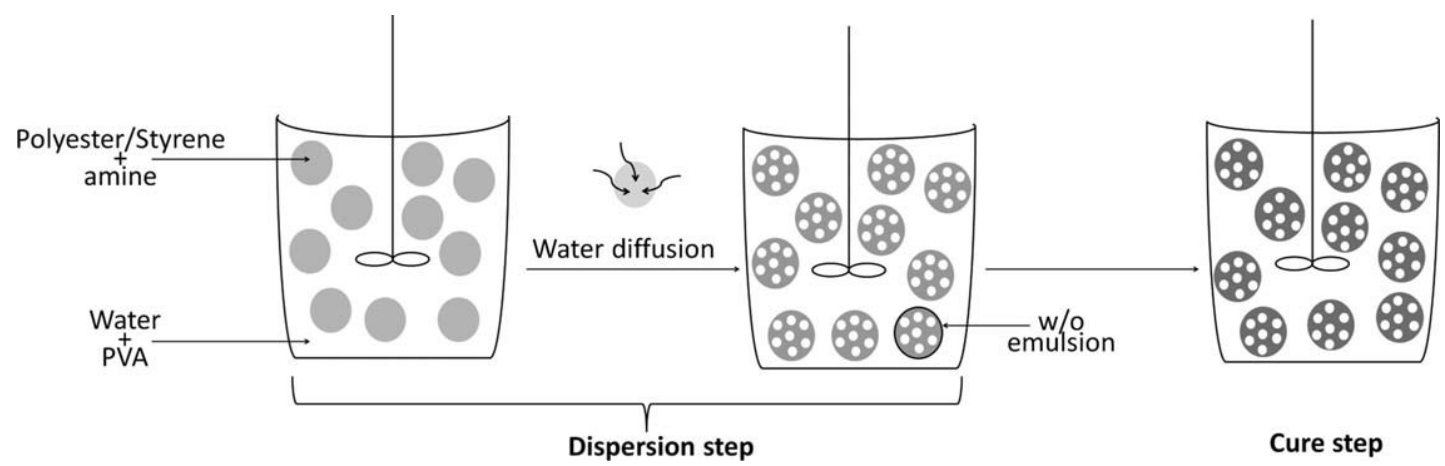

FIG. 1. Schematic representation of the MVPs synthesis process.

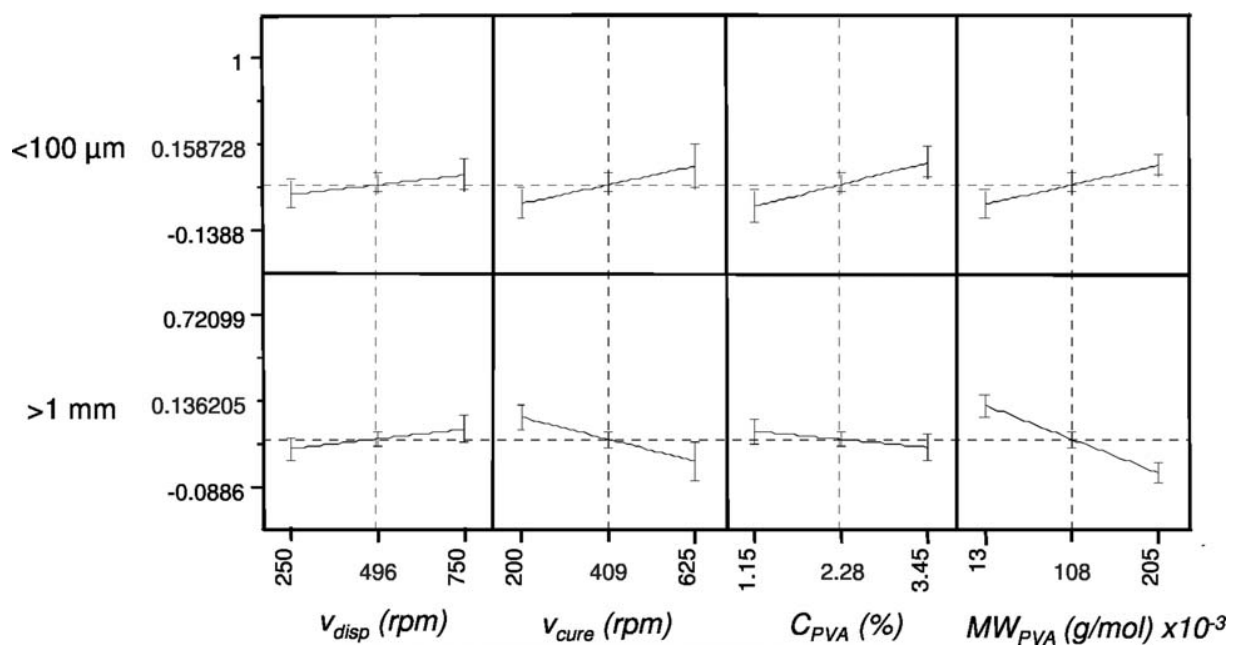

FIG. 2. Prediction profiles of the effects of the factor levels studied on particle size. 


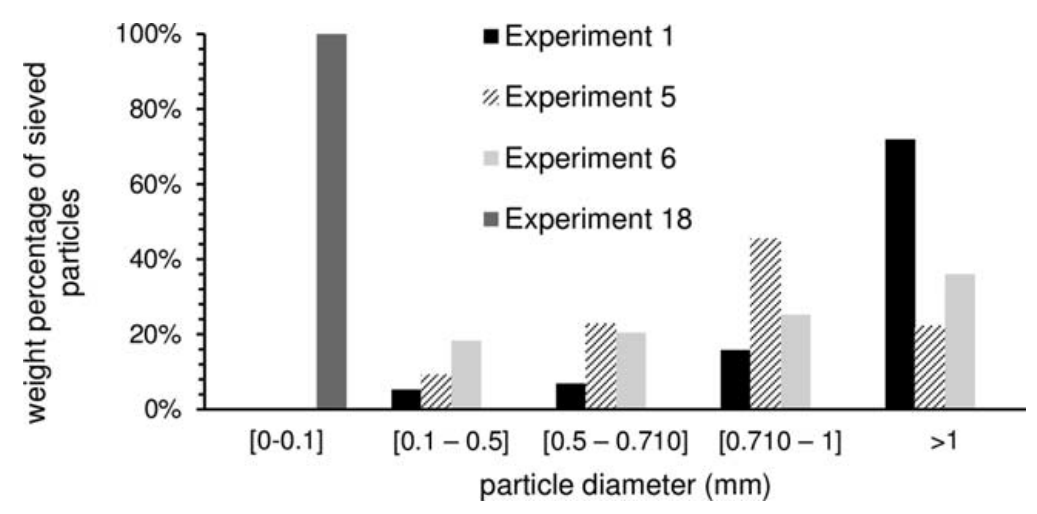

FIG. 3. Weight-based particle size distributions obtained by sieving for MVPs produced in Experiments 1, 5, 6, and 18.

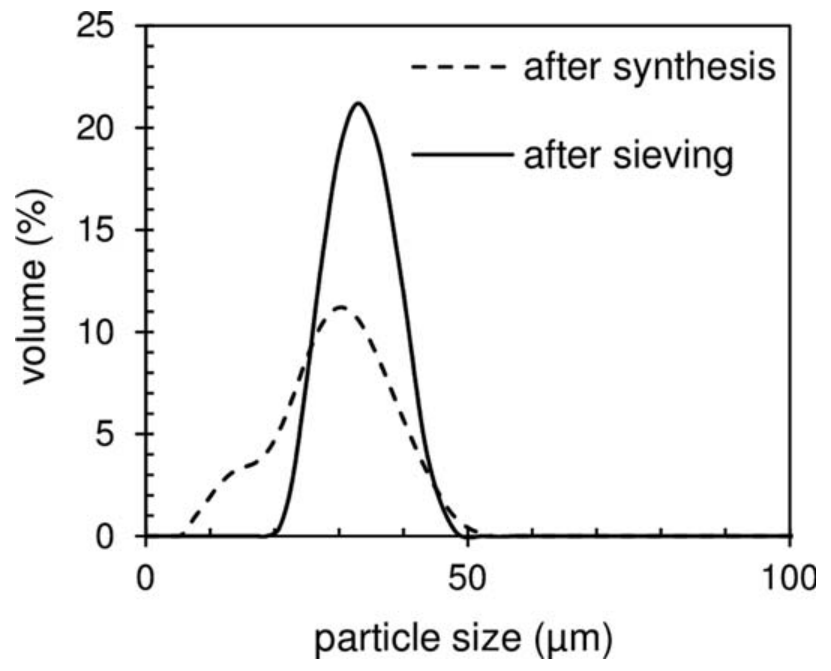

FIG. 4. Particle size distribution obtained by light scattering for MVPs produced in Experiment 18. 

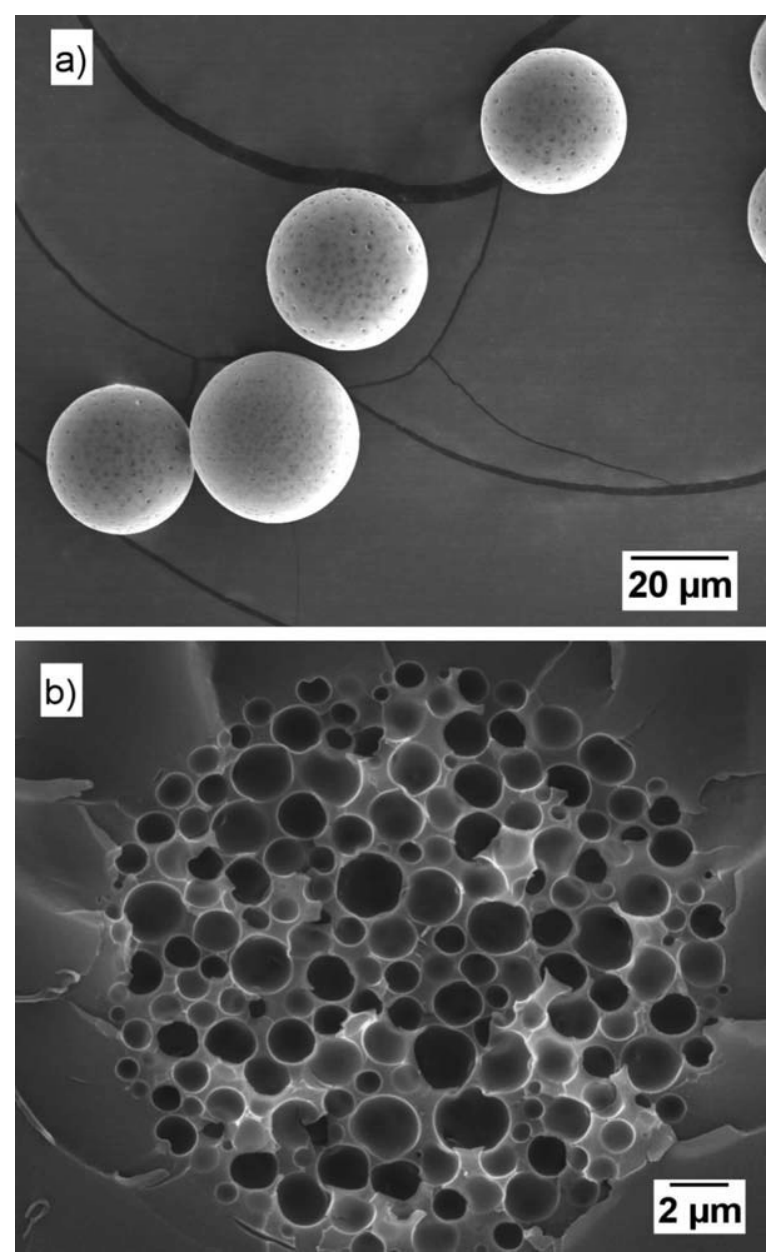

FIG. 5. SEM image of MVPs produced in Experiment 18: particle surface (magnification 4000x) (a) and after fracture of an epoxy-immobilized particle under liquid nitrogen, showing uniform interior vesiculation. 

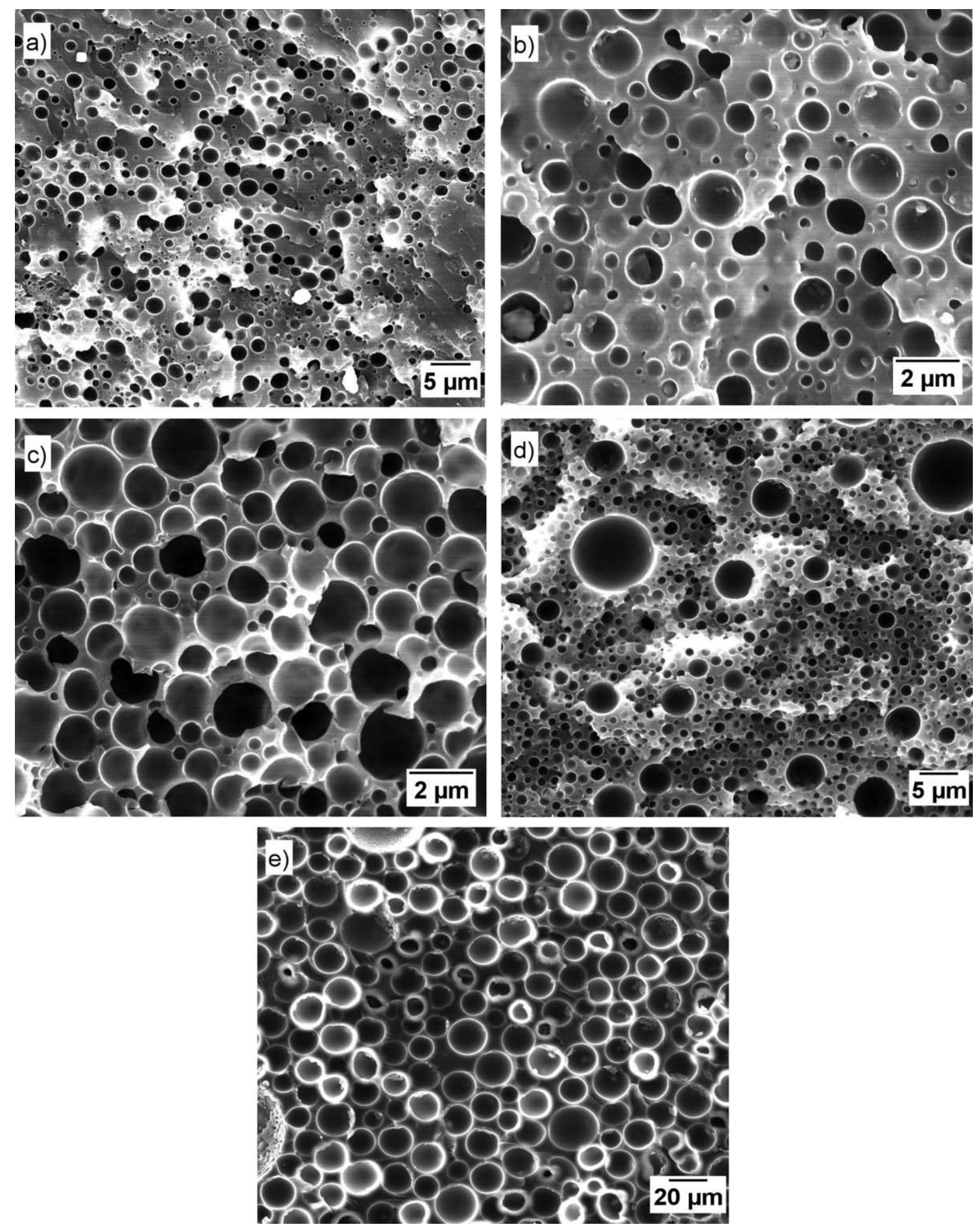

FIG. 6. SEM images of center region of MVPs produced under different dispersion stirring rates: (a) $200 \mathrm{rpm}$, (b) $600 \mathrm{rpm}$, (c) $750 \mathrm{rpm}$, (d) $1000 \mathrm{rpm}$, and (e) $1400 \mathrm{rpm}$. Particles were produced with PVA concentration of $2.3 \%$ and $\mathrm{MW}$ of $13,000 \mathrm{~g} / \mathrm{mol}$. After the dispersion step, particles were cured at $200 \mathrm{rpm}$ during $2 \mathrm{~h}$ at $60^{\circ} \mathrm{C}$ using $\mathrm{BPO}$ as an organic-soluble radical initiator. 


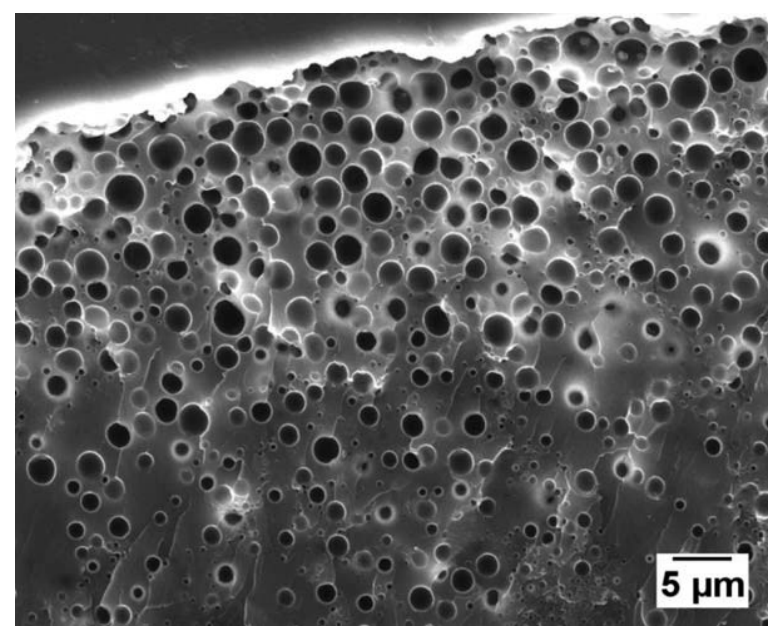

FIG. 7. SEM image of the periphery of a cured particle produced under $v$ disp $=200 \mathrm{rpm}$.

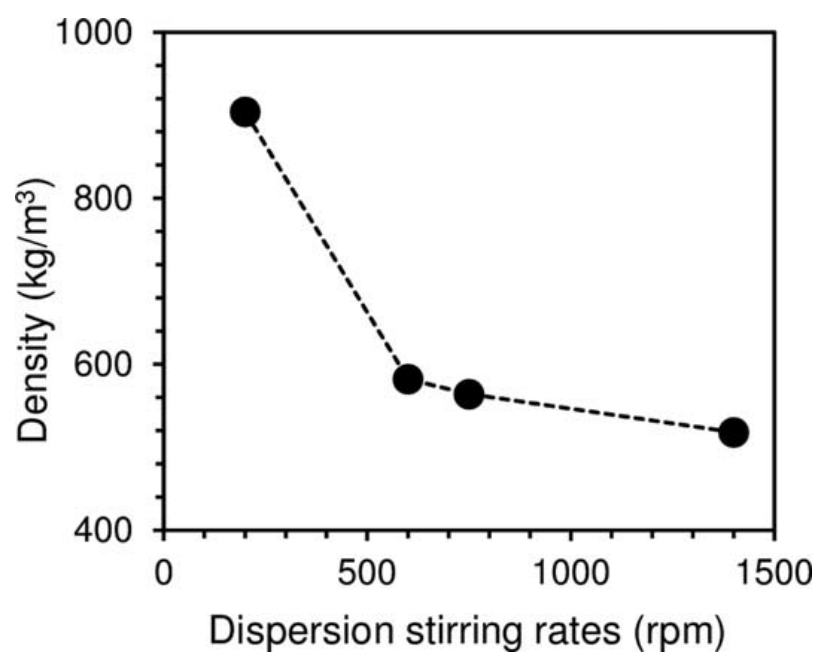

FIG. 8. Effect of dispersion stirring rates on MVPs apparent density.

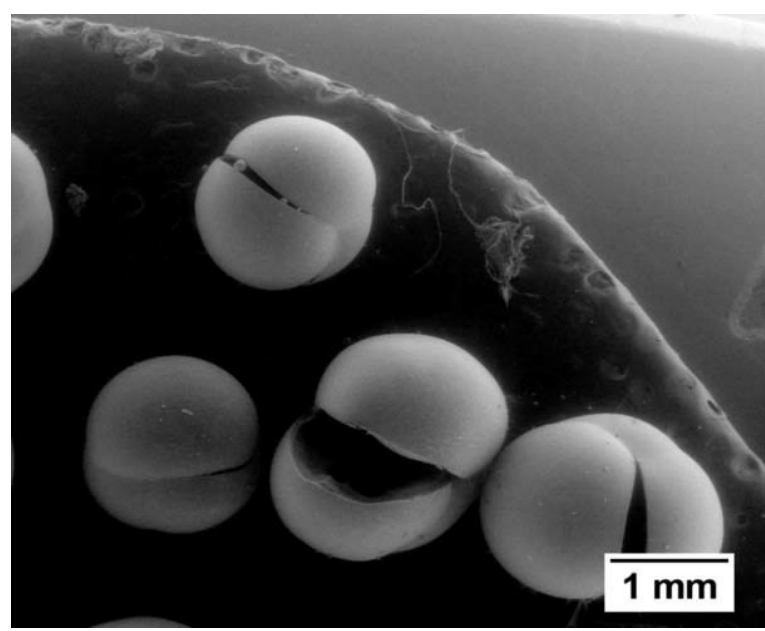

FIG. 9. SEM images of MVPs obtained after the drying process at $80^{\circ} \mathrm{C}$ for $2 \mathrm{~h}$ (magnification 40x). Particles were produced using cumene hydroperoxide as water-soluble initiator $($ MWPVA $=13,000 \mathrm{~g} / \mathrm{mol}, v$ disp1=750 rpm, during $20 \mathrm{~min}, v$ disp2=200 rpm, during 15 min and $v$ cure $=200 \mathrm{rpm}$, cure step took $2 \mathrm{~h}$ at $70^{\circ} \mathrm{C}$ ). 


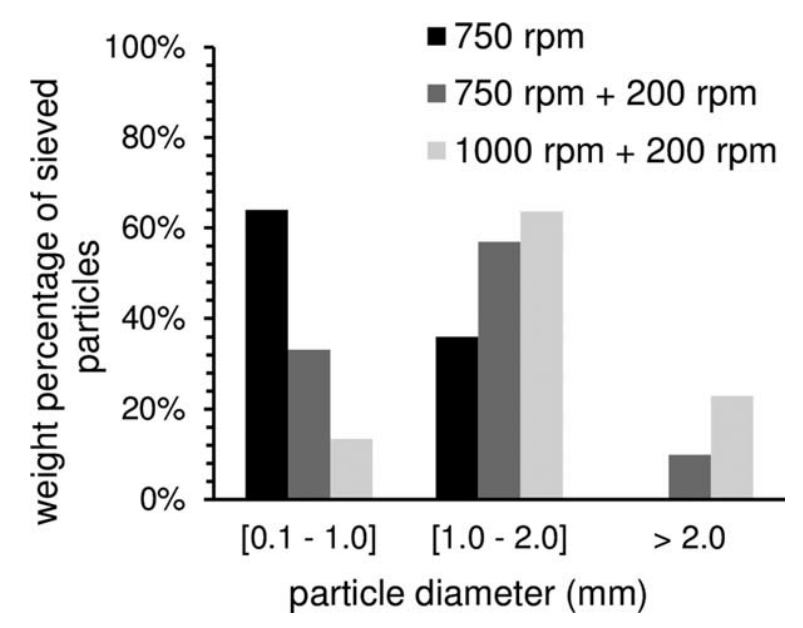

FIG. 10. Influence of the implementation of a second lower stirring rate during the dispersion step on the final particle size distribution. Particles were produced with PVA concentration of $2.3 \mathrm{wt} \%$ and molecular weight $13,000 \mathrm{~g} / \mathrm{mol}$, the first stirring rate was maintained for $20 \mathrm{~min}$ followed by $200 \mathrm{rpm}$ for $15 \mathrm{~min}$, and curing under the same stirring rate for $2 \mathrm{~h}$ at $60^{\circ} \mathrm{C}$.
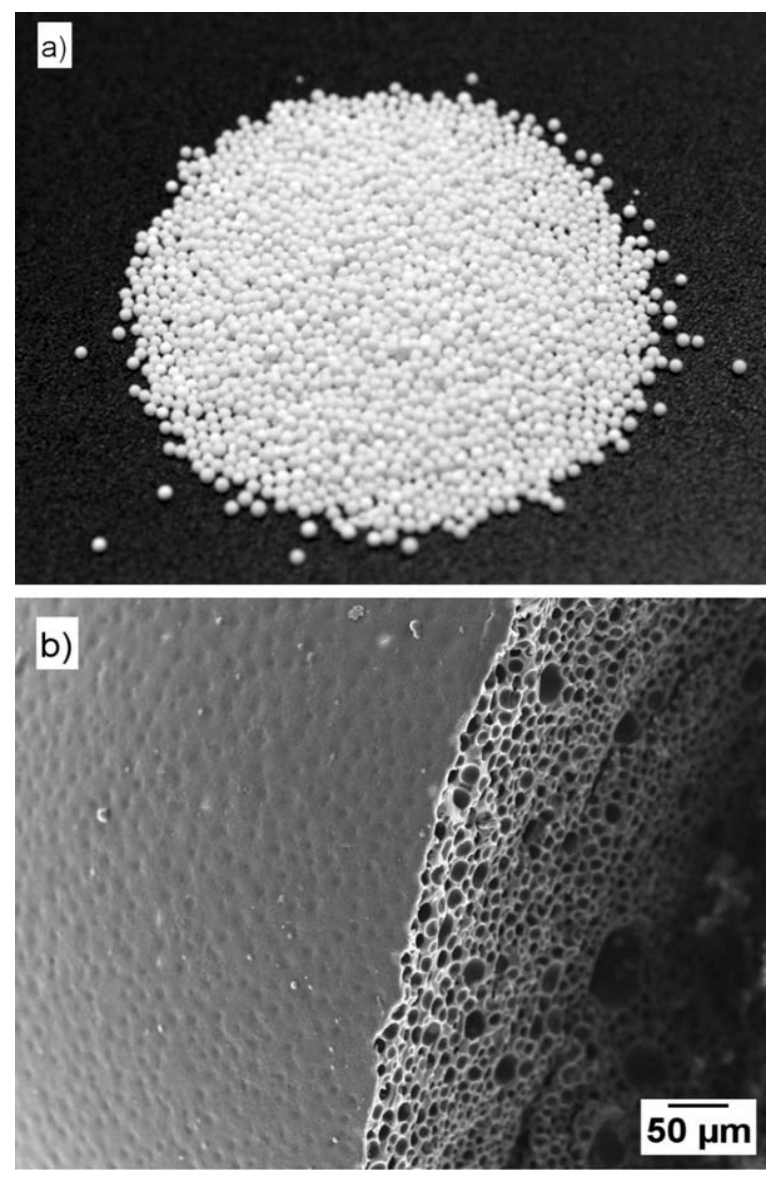

FIG. 11. MVPs produced with BPO as cure initiator, PVA concentration of $2.3 \mathrm{wt} \%$, and molecular weight 13,000 g/mol, vdisp1 $51000 \mathrm{rpm}$ (during $20 \mathrm{~min}$ ), vdisp2 (during $15 \mathrm{~min}$ ), cure temperature was $60^{\circ} \mathrm{C}$ during $2 \mathrm{~h}$, under a stirring rate of $200 \mathrm{rpm}$ (a), and SEM image after manual fracture, showing particle surface and uniform interior vesiculation (magnification 10,000x) (b). 
TABLE 1. Process variables (factors) and set levels.

\begin{tabular}{lccc}
\hline Factors & Level 1 & Level 2 & Level 3 \\
\hline$v_{\text {disp }}(\mathrm{rpm})$ & 250 & 500 & 750 \\
$v_{\text {cure }}(\mathrm{rpm})$ & 200 & 350 & 500 \\
$C_{\text {PVA }}(\%)$ & 1.15 & 2.3 & 3.45 \\
MW $_{\text {PVA }}(\mathrm{g} / \mathrm{mol})$ & 13,000 & 205,000 & \\
\hline
\end{tabular}

The factors studied were stirring rate during the dispersion step $\left(v_{\text {disp }}\right)$, stirring rate during the curing phase ( $\left.v_{\text {cure }}\right)$, PVA concentration $\left(C_{\mathrm{PVA}}\right)$, and PVA molecular weight $\left(\mathrm{MW}_{\mathrm{PVA}}\right)$. 
TABLE 2. L18 orthogonal array used for design of experiments, according to Taguchi method, and particle weight fractions obtained in each experiment.

\begin{tabular}{|c|c|c|c|c|c|c|}
\hline \multirow[b]{2}{*}{ Experiment } & \multicolumn{4}{|c|}{ Factors } & \multicolumn{2}{|c|}{ Performance parameters } \\
\hline & $v_{\text {disp }}(\mathrm{rpm})$ & $v_{\text {cure }}(\mathrm{rpm})$ & $C_{\mathrm{PVA}}(\%)$ & $\begin{array}{r}\mathrm{MW}_{\text {PVA }} \\
(\mathrm{g} / \mathrm{mol})\end{array}$ & $\begin{array}{c}\text { Particles } \\
\text { diameter }<100 \mu \mathrm{m}(\mathrm{wt} \%)\end{array}$ & $\begin{array}{c}\text { Particles } \\
\text { diameter }>1 \mathrm{~mm}(\mathrm{wt} \%)\end{array}$ \\
\hline 1 & 250 & 200 & 1.15 & 13,000 & 0 & 72 \\
\hline 2 & 500 & 350 & 1.15 & 13,000 & 0 & 17 \\
\hline 3 & 750 & 500 & 1.15 & 13,000 & 0 & 45 \\
\hline 4 & 250 & 350 & 2.3 & 13,000 & 0 & 3 \\
\hline 5 & 500 & 500 & 2.3 & 13,000 & 0 & 22 \\
\hline 6 & 750 & 200 & 2.3 & 13,000 & 0 & 36 \\
\hline 7 & 250 & 500 & 3.45 & 13,000 & 0 & 2 \\
\hline 8 & 500 & 200 & 3.45 & 13,000 & 0 & 46 \\
\hline 9 & 750 & 350 & 3.45 & 13,000 & 0 & 50 \\
\hline 10 & 250 & 500 & 1.15 & 205,000 & 0 & 72 \\
\hline 11 & 500 & 200 & 1.15 & 205,000 & 0 & 0 \\
\hline 12 & 750 & 350 & 1.15 & 205,000 & 1 & 0 \\
\hline 13 & 250 & 200 & 2.3 & 205,000 & 4 & 0 \\
\hline 14 & 500 & 350 & 2.3 & 205,000 & 0 & 0 \\
\hline 15 & 750 & 500 & 2.3 & 205,000 & 32 & 0 \\
\hline 16 & 250 & 350 & 3.45 & 205,000 & 32 & 0 \\
\hline 17 & 500 & 500 & 3.45 & 205,000 & 100 & 0 \\
\hline 18 & 750 & 200 & 3.45 & 205,000 & 100 & 0 \\
\hline
\end{tabular}


TABLE 3. ANOVA by factor using the mean: statistical significance level of the effects of the different factors on MVPs with diameter $<100 \mathrm{~mm}$.

\begin{tabular}{lccrrl}
\hline Factor & $\begin{array}{c}\text { Degrees } \\
\text { of freedom }\end{array}$ & $\begin{array}{c}\text { Sum of } \\
\text { squares }\end{array}$ & F ratio & p-value & $\begin{array}{c}\text { Significance } \\
\text { level }\end{array}$ \\
\hline$v_{\text {disp }}$ & 2 & 0.1253980 & 2.6043 & 0.1118 & NS \\
$v_{\text {cure }}$ & 2 & 0.2582725 & 5.3638 & 0.0240 & $*$ \\
$C_{\text {PVA }}$ & 2 & 0.6200788 & 12.8778 & 0.0007 & $* * *$ \\
MW $_{\text {PVA }}$ & 1 & 1.0152945 & 21.0857 & $<0.0001$ & $* * *$ \\
\hline
\end{tabular}

NS, not significant; *5\%;**1\%;***0.1\%.

TABLE 4. ANOVA by factor using the mean: statistical significance level of the effects of the different factors on MVPs with diameter larger than 1 mm.

\begin{tabular}{lccrrl}
\hline Factor & $\begin{array}{c}\text { Degrees of } \\
\text { freedom }\end{array}$ & $\begin{array}{c}\text { Sum of } \\
\text { squares }\end{array}$ & $F$ ratio & $p$-value & $\begin{array}{c}\text { Significance } \\
\text { level }\end{array}$ \\
\hline$v_{\text {disp }}(\mathrm{rpm})$ & 2 & 0.0703360 & 4.2407 & 0.0438 & $*$ \\
$v_{\text {cure }}(\mathrm{rpm})$ & 2 & 0.1808906 & 10.9064 & 0.0016 & $* * *$ \\
$C_{\text {PVA }}$ & 2 & 0.0418143 & 2.5211 & 0.1176 & $\mathrm{NS}$ \\
MW $_{\text {PVA }}$ & 1 & 1.5714778 & 94.7486 & $<0.0001$ & $* * *$ \\
\hline
\end{tabular}

NS, not significant; * 5\%;**1\%;***0.1\%. 